\title{
Angiomotin promotes breast cancer cell proliferation and invasion
}

\author{
MENG LV ${ }^{1 *}$, MEILING LV ${ }^{1 *}$, LING CHEN $^{1}$, TIANJIE QIN ${ }^{1}$, XIAO ZHANG $^{1}$, PEIJUN LIU $^{2}$ and JIN YANG ${ }^{1}$ \\ ${ }^{1}$ Department of Oncology and ${ }^{2}$ Center for Translational Medicine, The First Affiliated Hospital, \\ Xi'an Jiaotong University College of Medicine, Xi'an, Shaanxi 710061, P.R. China
}

Received November 25, 2014; Accepted January 21, 2015

DOI: 10.3892/or.2015.3780

\begin{abstract}
Angiomotin (Amot) is a multifunctional protein involved in endothelial cell migration and tube formation and angiogenesis. However, the biological role and molecular mechanism for the abnormal expression of Amot in breast cancer is poorly understood. The aim of the present study was to examine the function of and relationship between Amot and the Hippo-Yes-associated protein (YAP) pathway. The expression and location of Amot was examined in breast cancer tissues and cell lines using immunohistochemistry, real-time polymerase chain reaction analysis (RT-PCR), western blotting and immunofluorescence. ANOVA, Student's t-test, Wilcoxon and Chi-square tests were utilized to determine the association of Amot expression with clinically relevant parameters. Stable Amot knockdown MCF-7 cells (MCF-7 Amot KD) were generated to investigate the impact of Amot downregulation on the growth and invasion of MCF-7 cells in vitro. Western blotting was applied to detect the expression of the Hippo-YAP pathway protein in the MCF-7 cells. It was observed that Amot was highly expressed in breast cancer tissues, but weakly expressed in adjacent non-cancerous tissues. Additionally, the expression level of Amot was correlated with that of $\mathrm{Ki}-67$. In MCF-7 cells, Amot downregulation resulted in a significant decrease of cell proliferation and invasiveness. Following Amot knockdown in MCF-7 cells, the expression of YAP, YAP/TAZ and LATS1 was decreased. In particular, the expression of YAP was markedly reduced in the nucleoprotein. The results suggested that Amot was highly expressed in breast cancer tissues and was important in the
\end{abstract}

Correspondence to: Professor Jin Yang, Department of Oncology, The First Affiliated Hospital, Xi'an Jiaotong University College of Medicine, 277 West Yanta Road, Xi'an, Shaanxi 710061, P.R. China E-mail: dryangjinla@aliyun.com

Professor Peijun Liu, Center for Translational Medicine, The First Affiliated Hospital, Xi'an Jiaotong University College of Medicine, 277 West Yanta Road, Xi'an, Shaanxi 710061, P.R. China

E-mail: liupeijun@mail.xjtu.edu.cn

*Contributed equally

Key words: angiomotin, breast cancer, proliferation, invasion, Hippo-YAP promotion of breast cancer cell proliferation and invasion. In addition, there was a more intimate connection between Amot and Hippo-YAP pathway.

\section{Introduction}

Breast cancer is a common malignant tumor that occurs in women. Approximately 1.2 million women suffer from, and $\sim 500,000$ succumb to breast cancer annually. According to the American Cancer Society (ACS), the incidence and mortality of breast cancer ranked the first and second of all female cancer types in America in 2014, accounting for 29 and $15 \%$, respectively (1). Approximately $50 \%$ of the breast cancer cases and $60 \%$ of the deaths are estimated to occur in economically developing countries (2). A significant reduction in the mortality of breast cancer patients worldwide has been observed in the past 20 years. This reduction has been largely due to improvement in the early detection and development of more effective adjuvant therapies (3). In addition, therapies have been developed to be specifically tailored to targeting each molecular subtype of breast cancer. These therapies include human epidermal growth factor receptor-2 (HER2)-targeting agents for HER2-overexpressing tumors, aromatase inhibitors, third-generation hormonal therapies for hormone-sensitive disease, and poly(ADP-ribose) polymerase (PARP) inhibitors for BRCA1-deficient and triple-negative breast cancers (4). Nevertheless, a large number of women with breast cancer experience relapse. Therefore, identification of more effective treatment targets is needed in breast cancer research.

Angiomotin (Amot) was first identified from its ability to bind to angiostatin using a yeast two-hybrid screen (5). Amot is characterized by a conserved coiled-coil domain and a C-terminal PDZ-binding motif (6) and is expressed as two different isoforms, p80-Amot and p130-Amot. Compared with p80-Amot, p130-Amot contains an extended N-terminal domain $(7,8)$. It was previously reported that Amot regulates endothelial cell migration, tube formation and is important in angiogenesis (6,9-13). Amot is also involved in regulating permeability and the movement of epithelial cells in tight junctions $(6,9,10)$. Using RT-PCR Jiang et al (14) found that Amot transcript was significantly highly expressed in human breast cancer tissues, particularly in highly invasive and metastatic tumor tissues, when compared with its expression in normal mammary tissues. However, the role and mechanism of the abnormal expression of Amot in breast cancer remain to be elucidated. 
To evaluate the function and mechanism of Amot, the expression and location of Amot in breast cancer, adjacent non-cancerous tissues, breast cancer and breast epithelium cell lines, were first determined using western blotting, RT-PCR and immunofluorescence. shRNA was applied to block and silence the expression of Amot in the breast cancer cell line and the effect of the silenced Amot on the biological behavior of breast cancer cell line was studied. The results showed that breast cancer tissues had a significantly increased Amot protein level, when compared with adjacent non-cancerous tissues and the expression level of Amot was closely correlated with the expression level of Ki-67 $(\mathrm{P}<0.01)$. We also observed that Amot downregulation resulted in a significant decrease in cell proliferation, cell invasiveness and migration in vitro and was closely associated with the Hippo-YAP pathway which regulates cell proliferation during development, tissue regeneration and carcinogenesis. Thus, Amot acts as a potential tumor promoter.

\section{Materials and methods}

Patients and tissue samples. A total of 242 breast cancer tissue samples and 92 adjacent non-cancerous tissue samples were obtained from the First Affiliated Hospital of Xi'an Jiaotong University College of Medicine and Shanghai Outdo Biotech Co. Ltd. (Shanghai, China). The clinicopathological tumornode-metastasis (TNM) staging was stage I for 16 cases, stage II for 139 cases and stage III-IV for 81 cases. Clinically relevant parameters are presented in Table I. The pathological types of all the specimens were confirmed by independent pathologists. No patients received radiotherapy or chemotherapy prior to surgery. TNM stages were assigned using the 2010 Union for International Cancer Control (UICC) criteria. The study was approved by the Human Ethics Committee of the First Affiliated Hospital, College of Medicine of Xi'an Jiaotong University. Informed consent was obtained from each patient. All of the specimens were fixed in $10 \%$ buffered formalin solution and embedded in paraffin.

Immunohistochemistry. Fixed tumor tissue samples were sectioned, deparaffinized, rehydrated and subjected to heat-induced antigen retrieval in EDTA buffer $(1.0 \mathrm{mM}$, $\mathrm{pH} 8.0$ ) for $10 \mathrm{~min}$ in a microwave oven. The samples were blocked with $3 \%$ hydrogen peroxide. After being blocked with $1 \%$ bovine serum albumin (BSA), the sections were incubated overnight at $4^{\circ} \mathrm{C}$ with a primary antibody specific for Amot (Genemed Synthesis Inc., San Antonio, TX, USA). Control sections were incubated with an isotype-matched polypeptide control antibody and phosphate-buffered saline (PBS). Subsequently, the sections were incubated with HRP-conjugated secondary antibody for $30 \mathrm{~min}$. The sections were stained with 3,3'-diaminobenzidine, and then counterstained with hematoxylin and examined under a microscope (Olympus CX21; Tokyo, Japan). To evaluate Amot protein expression, the staining intensity was graded as 0 for no staining; 1 for weak staining; 2 for moderate staining; or 3 for strong staining. The extent of staining was scored according to the percentage of positively stained cells as follows: $0(\leq 5 \%)$, $1(6-25 \%), 2(26-50 \%), 3(51-75 \%)$ and $4(76-100 \%)$. The number of positively stained cells was determined by counting
Table I. Amot expression in breast cancer and adjacent non-cancerous tissues.

\begin{tabular}{lcccc}
\hline Group & $\begin{array}{c}\text { Negative } \\
\text { expression } \\
(\%)\end{array}$ & $\begin{array}{c}\text { Weak } \\
\text { expression } \\
(\%)\end{array}$ & $\begin{array}{c}\text { Moderate } \\
\text { expression } \\
(\%)\end{array}$ & $\begin{array}{c}\text { Strong } \\
\text { expression } \\
(\%)\end{array}$ \\
\hline $\begin{array}{l}\text { Cancer } \\
\text { tissues }\end{array}$ & $37(15.3)$ & $54(22.3)$ & $106(43.8)$ & $45(18.6)$ \\
$\begin{array}{l}\text { Non- } \\
\text { cancerous } \\
\text { tissues }\end{array}$ & $80(87)$ & $8(8.6)$ & $4(4.3)$ & $0(0)$ \\
$\begin{array}{l}\text { Total } \\
\mathrm{H}\end{array}$ & 117 & 62 & 110 & 45 \\
$\mathrm{P}$ & 129.4 & & & \\
\hline
\end{tabular}

${ }^{\mathrm{a}} \mathrm{P}<0.001$. Amot, Angiomotin.

cells from 10 random fields at x400 magnification. The final immunohistochemical staining score was obtained by multiplying the staining intensity and the extent of staining (negative expression, scores 0-2; weak expression, scores 3-5, moderate expression, scores 6-9; and strong expression, scores 10-12).

Antibodies. The Amot antibody was produced by Genemed Synthesis Inc. The synthetic peptide sequence (C+LVKSSSKRE ALEKAMR and C-KTPIQILGQEPDAEMVEYLI) was conjugated to keyhole limpet hemocyanin (KLH) for immunizations. GAPDH and rabbit flag were purchased from Santa Cruz Biotechnology, Inc. (Santa Cruz, CA, USA). YAP, YAP/ TAZ, LATS1, MOB, MST1 and SAV1 were purchased from Cell Signaling Technology (Danvers, MA, USA).

Cell culture and lentiviral transfection. The MCF-7, T-47D, BT-474, MDA-MB-453, MDA-MB-231 breast cancer cell lines and MCF-10A breast epithelial cell line were purchased from the American Type Culture Collection (ATCC; Manassas, VA, USA). MCF-10A cells were cultured in Dulbecco's modified Eagle's medium (DMEM)/F12 (Invitrogen, Carlsbad, CA, USA) supplemented with 5\% horse serum (HyClone, Logan, UT, USA), $1 \%$ penicillin/streptomycin, $0.5 \mu \mathrm{g} / \mathrm{ml}$ hydrocortisone, $10 \mu \mathrm{g} / \mathrm{ml}$ insulin (both from Sigma, Santa Clara, $\mathrm{CA}, \mathrm{USA}$ ) and $20 \mathrm{ng} / \mathrm{ml}$ recombinant human EGF (Invitrogen). MCF-7, T47D and MDA-MB-435 breast cancer cell lines were cultured in DMEM supplemented with $10 \%$ fetal bovine serum (FBS) (both from HyClone). BT474 was cultured in RPMI-1640 medium supplemented with 10\% FBS (both from HyClone). All the cell cultures were maintained at $37^{\circ} \mathrm{C}$ in a humidified atmosphere containing $5 \% \mathrm{CO}_{2}$.

Lentivirus vectors for human Amot small hairpin RNA (shRNA) encoding a green fluorescent protein (GFP) and a puromycin-resistant gene were constructed, packed and purified by GeneChem Co., Ltd. (Shanghai, China). The RNA interference sequences used were: shAMOT (8489-1R), 5'-TGCAGAGATGGTGGAATAT-3'; shAMOT (8491-2R) 5'-ACACATCGAAATCCGAGAT-3'; negative control: shNC, 5'-TTCTCCGAATGTGTCACGT-3'. 
MCF-7 cells were transfected with lentivirus according to the manufacturer's instructions (GeneChem Co., Ltd.). For transfection, the lentiviruses mixed with medium containing Polybrene were added to the cells at the confluence of $30-40 \%$. After $8 \mathrm{~h}$ of transfection, the medium was replaced by fresh DMEM medium containing $10 \%$ FBS. Seventy-two hours after transfection, the cells were selected with $3.5 \mu \mathrm{g} / \mathrm{ml}$ puromycin for 2 weeks. Amot knockdown was verified by western blot analysis. The cells were divided into three groups: CON (control; the uninfected breast cancer cells); KD (knockdown; cells transfected with the Amot shRNA lentivirus); NC (negative control; cells transfected with the mock control lentivirus).

Real-time polymerase chain reaction analysis. Total mRNA was extracted using the Fast 200 reagent (Pioneer Biotechnology Inc., Shaanxi, China) and reverse transcription was performed using an RT-PCR kit (Takara, Dalian, China). Complementary DNA synthesis was conducted using a SYBR ExScript RT-PCR kit (Takara) according to the manufacturer's instructions. RT-PCR was conducted using the iQ5 Multicolor Real-Time PCR Detection System (Bio-Rad, Hercules, CA, USA) and SYBR Premix Ex Taq ${ }^{\mathrm{TM}}$ II (Takara). The primer sequences used for amplification were: Amot 5'-CCAGAATAT CCCTTCAAG-3' and 5'-GAGTTCCTGGCTGACAAT-3', GAPDH: 5'-CTCCTCCACCTTTGACGCTG-3' and 5'-TCCT CTTGTGCTCTTGCTGG-3'. GAPDH was applied as the internal housekeeping gene control. Each reaction was performed in a final volume of $10 \mu \mathrm{l}$ containing $1.0 \mu \mathrm{l}$ of appropriately diluted cDNA, $0.4 \mu \mathrm{l}(10 \mu \mathrm{M})$ of forward and reverse primers specific for human Amot or GAPDH, $5 \mu \mathrm{l}$ of SYBR Premix Ex Taq and $3.2 \mu 1$ of water. The PCR consisted of $1 \mathrm{~min}$ at $95^{\circ} \mathrm{C}$ followed by 40 cycles of denaturation for $15 \mathrm{sec}$ at $95^{\circ} \mathrm{C}$, annealing for $15 \mathrm{sec}$ at $55^{\circ} \mathrm{C}$ and a primer extension for $45 \mathrm{sec}$ at $72^{\circ} \mathrm{C}$. The ${ }^{\Delta \Delta} \mathrm{Ct}$ method was used for the relative quantification of Amot expression.

Western blotting. Cells were lysed with RIPA buffer [50 mmol/1 Tris (pH 7.5), $100 \mathrm{mmol} / 1 \mathrm{NaCl}, 1 \mathrm{mmol} / 1$ EDTA, $0.5 \%$ NP40, $0.5 \%$ Triton X-100, $2.5 \mathrm{mmol} / 1$ sodium orthovanadate, $10 \mu \mathrm{l} / \mathrm{ml}$ protease inhibitor cocktail and $1 \mathrm{mmol} / 1$ PMSF] by incubating for $20 \mathrm{~min}$ at $4^{\circ} \mathrm{C}$. Protein concentrations were determined by a BCA assay (Pierce, Rockford, IL, USA). Equal amounts of the cell lysate protein were subjected to $10 \%$ SDS-PAGE, transferred to nitrocellulose membranes (Millipore, Boston, MA, USA), blocked with 5\% non-fat dry milk in Tris-buffered saline with Tween-20 for $1 \mathrm{~h}$, and incubated with the indicated antibody overnight at $4^{\circ} \mathrm{C}$. The reactive bands were developed by chemiluminescence with the luminol reagent (Millipore). The blots were re-probed with $G A P D H$ antibody as a loading control.

Immunofluorescent staining. MCF-7, BT-474 and MDA-MB231 cells were cultured on coverslips to the appropriate densities. The cells were fixed with $4 \%$ paraformaldehyde solution for $10 \mathrm{~min}$ at room temperature, washed three time with PBST and then permeabilized with $0.1 \%$ Triton X-100 for 10 min. The slides were blocked with 5\% BSA and $10 \%$ horse serum in PBST for $1 \mathrm{~h}$ at room temperature and incubated with antibodies against Amot $(1: 100)$ overnight at $4^{\circ} \mathrm{C}$. After being washed with PBS, cells were incubated with IgG-HRP secondary antibody (1:100; ZhongShan Jinqiao Biological Company, Peking, China) for $1 \mathrm{~h}$ at RT. The cells were then washed three times and visualized using a laser scanning confocal microscope (Leica, Germany).

Cell proliferation assay. Cells were seeded in $1 \%$ gelatin-coated 96 -well plates with $5 \times 10^{3}$ cells/well. Relative cell numbers were quantified each day using the 3-(4,5-dimethylthiazol2-yl)-2,5-diphenyltetrazolium bromide (MTT; Sigma) assay. The absorbance was measured at $492 \mathrm{~nm}$ using a multifunction microplate reader (POLARstar OPTIMA, Germany).

Plate colony formation assay. The cells were trypsinized and resuspended in DMEM containing 10\% FBS. One handred cells were cultured in 6-well plates until visible cell colonies were formed. After the cells were fixed and stained, the number of cell colonies was counted under a microscope (Leica, Germany).

5-Bromodeoxyuridine (Brdu) incorporation assay. Brdu assay was used to detect the variation of the $S$ phase of breast cancer cells and evaluate the effect of Amot downregulation on cell proliferation. Brdu $(10 \mu \mathrm{mol} / \mathrm{l})$ was added to the medium, cultured in a constant-temperature incubator for $40 \mathrm{~min}$, and fixed by $35 \%$ ethanol at $4^{\circ} \mathrm{C}$ for $1-2 \mathrm{~h}$. Then cells were resuspended in $2 \mathrm{~N} \mathrm{Hcl}$ and $0.1 \mathrm{M}$ sodium borate successively. After centrifugation at $800 \mathrm{x} \mathrm{g}$ for $5 \mathrm{~min}$, the supernatant was decanted and the cells were cultured in $5 \mu \mathrm{l}$ anti-Brdu antibody (BD Biosciences, Bedford, MA, USA) and $45 \mu 1$ PBS (containing $0.5 \%$ Tween-20 $+0.5 \% \mathrm{BSA}$ ) at room temperature for $30 \mathrm{~min}$ away from light. The $\mathrm{S}$ phase of the cells were observed by flow cytometry (BD Biosciences).

Wound-healing assay. The cells were seeded in 6-well plates and cultured with DMEM containing 10\% FBS until the cells reached subconfluence. Following removal of the culture medium, a monolayer of the sub-confluent cells was scratched with a $200 \mu$ l pipette tip to create a wound area. The wounded monolayer was washed with PBS twice and cultured in FBS-free medium or $2 \%$ FBS medium for $48 \mathrm{~h}$. Cell migration into the wound area was monitored by inverted microscopy, and photographed at the indicated time points until the wound was completely closed.

Cell migration and invasion assays. Migration and invasion assays were performed using the BioCoat cell migration chamber (BD Biosciences), which consists of a 24-well companion plate with cell culture inserts containing a filter with $8-\mu \mathrm{m}$-diameter pores. The Transwell for the invasion assay was coated with Matrigel (1:3 dilution with DMEM free of serum; BD Biosciences). The cells were trypsinized and suspended with DMEM without FBS at $2 \times 10^{5} / \mathrm{ml}$ for the migration assay and with DMEM without FBS at $2 \times 10^{6} / \mathrm{ml}$ for the invasion assay. Cell suspension (100 $\mu \mathrm{l})$ was added to the upper well and $600 \mu \mathrm{l}$ DMEM medium containing $10 \%$ FBS was added to the lower well. Cells in the wells were incubated in $5 \% \mathrm{CO}_{2}$ at $37^{\circ} \mathrm{C}$ for $24 \mathrm{~h}$ for the migration assay and $48 \mathrm{~h}$ for the invasion assay. After incubation, cells in the upper wells were gently removed by scrubbing, fixed in $95 \%$ ethanol for $15 \mathrm{~min}$ and stained with $0.4 \%$ crystal violet for 
$\mathbf{A}$

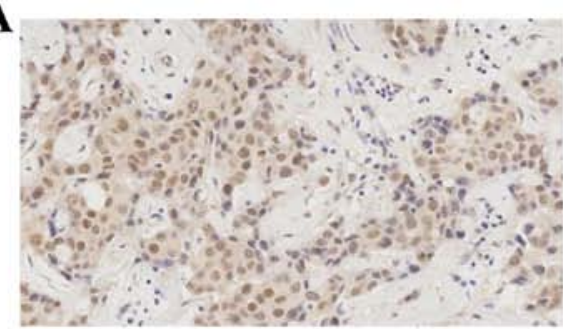

C

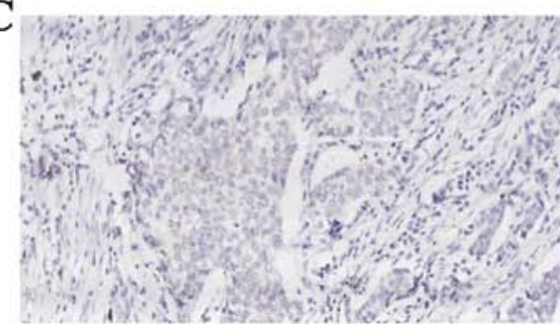

$\mathbf{E}$
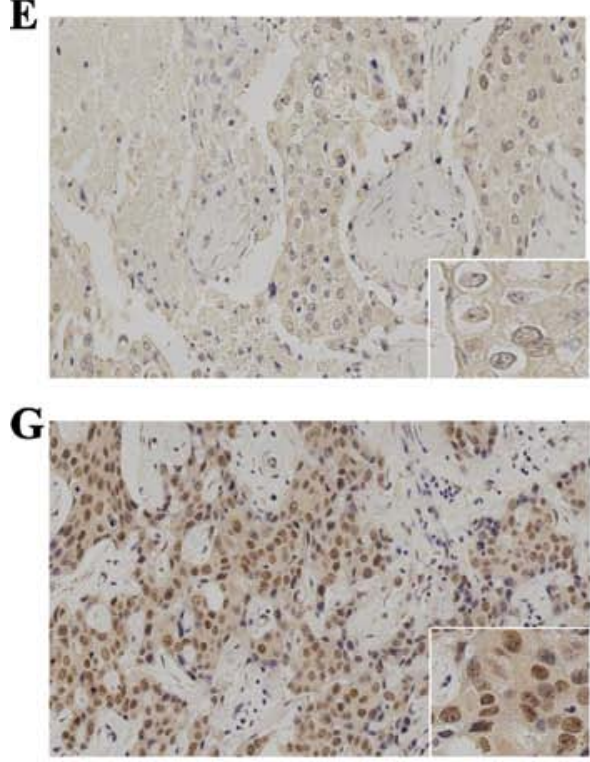

B

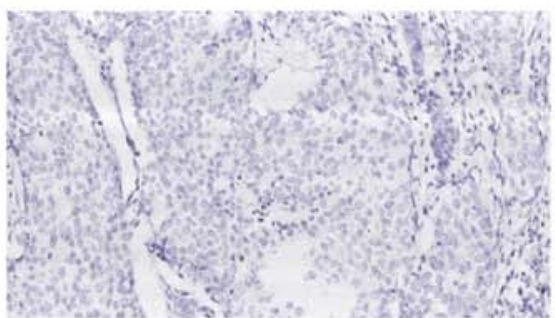

D

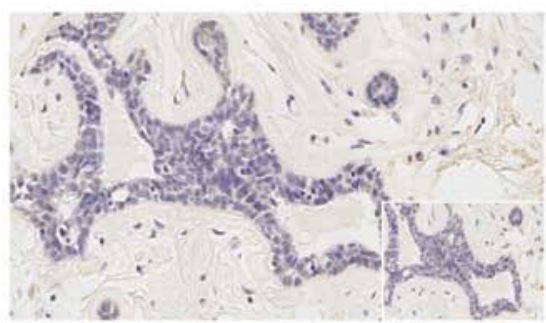

F
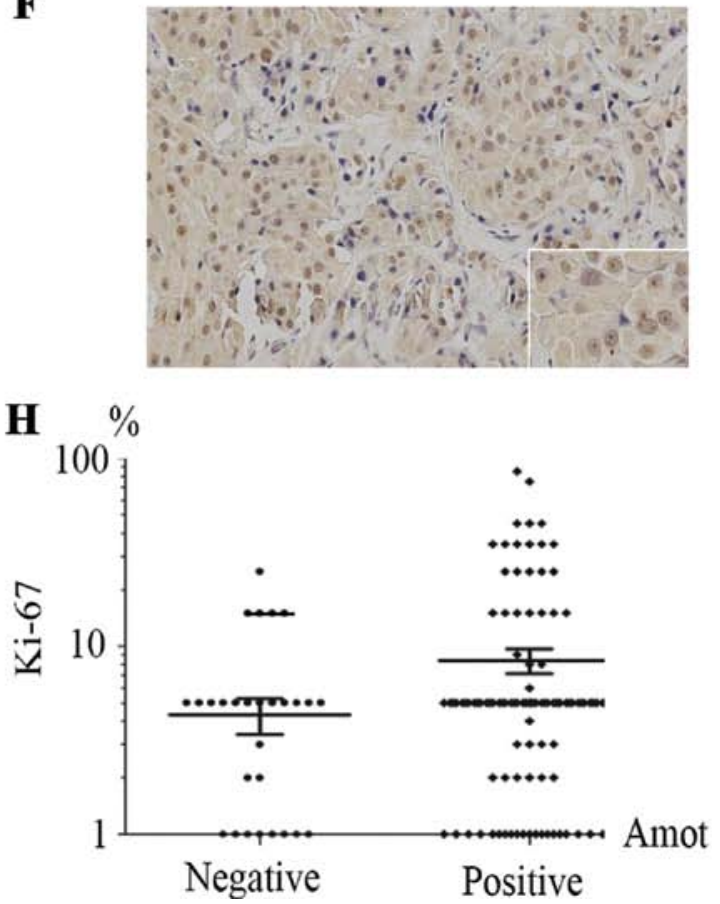

Figure 1. Expression and localization of Angiomotin (Amot) in breast cancer tissues. (A-C) Detection of antibody specificity in breast cancer tissues by immunohistochemistry (magnification, x200): (A) Amot antibody group; (B) Amot antibody mixed with enclosed peptide group; (C) PBS (negative control) group. (D-G) Immunohistochemical staining of Amot in adjacent non-cancerous tissues (D), and breast cancer tissues (E-G) (magnification, x200): (D) negative expression (scores 0-2); (E) weak expression (scores 3-5); (F) moderate expression (scores 6-9); (G) strong expression (scores 10-12). Each inset showed images captured at a magnification of $\mathrm{x} 400$. (H) Association of Amot and Ki-67. Amot-positive tumors have higher Ki-67.

30 min.Invasive or migrated cells were subsequently photographed with a microscope.

Statistical analysis. Statistical analysis was performed using SPSS 13.0 (Chicago, IL, USA). Data were presented as the mean \pm SD for at least three replicates for each group. Statistical differences between groups were determined using the ANOVA, Student's t-test, Wilcoxon and Chi-square tests. $\mathrm{P}<0.05$ was considered to indicate a statistically significant result.

\section{Results}

Amot expression and localization in breast cancer and adjacent non-cancerous tissues. To verify the specificity of the
Amot antibody, immunohistochemistry of breast cancer tissues was performed using Amot antibody, polypeptide-enclosed Amot antibody and PBS (negative control), respectively. The results showed that Amot was highly expressed in breast cancer tissues with Amot antibody, and negatively expressed in breast cancer tissues with polypeptide-enclosed Amot antibody or PBS (Fig. 1A-C). The results suggested that Amot antibody was specific and sensitive.

Immunohistochemistry was performed on 242 breast cancer and 92 adjacent non-cancerous tissues, to assess the expression and localization of Amot. The results showed that Amot was expressed in 205 breast cancer and 12 adjacent non-cancerous tissues. Amot was highly expressed in breast cancer tissues, but weakly expressed in adjacent non-cancerous 
tissues. The difference was statistically significant $(\mathrm{P}<0.001$; Table I). Notably, Amot expression was observed in the nucleus and cytoplasm of breast cancer tissues. In particular, Amot was strongly positively expressed in the nucleus. However, Amot showed a weak positive expression in the cytoplasm of adjacent non-cancerous tissues (Fig. 1D-G). We examined whether the strong positive expression of Amot in breast cancer tissues was connected with the clinically relevant parameters including age, tumor size, clinical stage, pathological grade, local lymph node status, the expression of ER, PR, Her-2 and Ki-67 (Table II). We found that the expression level of Amot was increased in specimens from patients with a high level of $\mathrm{Ki}-67$, which is an immunohistochemical proliferation marker in many types of cancer (Fig. 1H; Table II; P $<0.01$ ). This finding indicated that Amot was significantly correlated with cell proliferation and invasion.

Amot expression and localization in breast cancer cell lines. The expression of Amot in MCF-7, T-47D, BT-474, MDA-MB453 and MDA-MB-231 breast cancer cell lines and the MCF-10A breast epithelial cell line was detected by western blotting and RT-PCR. Our findings showed that Amot mRNA and protein were expressed in all the breast cancer cell lines, while the Amot expression level was significantly higher in MCF-7 cells than in the remaining cell lines (Fig. 2A and C). The specificity of the Amot antibody was verified by western blotting (Fig. 2B). Notably, Amot was expressed in the nucleus and cytoplasm of breast cancer cells. In particular, a strong positive Amot expression was observed in the nucleus. The localization of Amot in breast cancer cell lines was similar to those in breast cancer tissues (Fig. 2D).

Amot downregulation decreased proliferative, invasive and metastatic capacity of MCF-7 cells in vitro. The Amot protein expression was effectively suppressed in MCF-7 cells by the shAmot lentivirus (Fig. 3A). We then conducted MTT assay, plate colony formation and BrdU incorporation assay to estimate the effect of Amot silencing on cell proliferation. MTT assay showed that compared to CON and $\mathrm{NC}$ cells, the cells in the Amot knockdown group grew gradually, with decreased cell viability (Fig. 3B). The plate colony formation assay demonstrated that in MCF-7 Amot KD cells, the number of colonies were reduced significantly (Fig. $3 \mathrm{C}$ and $\mathrm{D} ; \mathrm{P}<0.001$ ). BrdU incorporation assay further verified the above findings. The Amot knockdown cells exhibited a significant decrease in the percentage of S-phase cells, when compared with the $\mathrm{CON}$ and NC cells (Fig. 3E and F; $\mathrm{P}<0.001$ ). The above results consistently suggested that Amot downregulation inhibited MCF-7 cell proliferation.

The effect of Amot downregulation on invasion and metastasis was explored using wound healing and Transwell assays. In the absence of the serum, cell migration showed no significant change between the Amot KD and NC groups. Of note, the NC cells exhibited characteristic morphological changes of apoptosis after the serum was decanted $24 \mathrm{~h}$, while the Amot knockdown cells were allowed to grow in the serum-free medium. In the presence of $2 \%$ FBS, the migration of the Amot knockdown cells was slower than that of the NC cells (Fig. 4A). Transwell assay was conducted to further confirm the abovementioned results. It was found that
Table II. The relationship between the expression of Amot protein and clinicopathological factors.

\begin{tabular}{|c|c|c|c|c|c|}
\hline \multirow[b]{2}{*}{ Variables } & \multirow[b]{2}{*}{$\mathrm{N}$} & \multicolumn{2}{|c|}{ Expression level } & \multirow[b]{2}{*}{$\chi^{2}$} & \multirow[b]{2}{*}{ P-value } \\
\hline & & Neg. & Pos. & & \\
\hline Age (years) & & & & 0.000 & 1.000 \\
\hline$<35$ & 11 & 2 & 9 & & \\
\hline$\geq 35$ & 229 & 36 & 19 & & \\
\hline Missing $^{\mathrm{a}}$ & 2 & & & & \\
\hline $\begin{array}{l}\text { Histological } \\
\text { grade }\end{array}$ & & & & 2.424 & 0.298 \\
\hline 1 & 18 & 5 & 13 & & \\
\hline 2 & 184 & 26 & 158 & & \\
\hline 3 & 40 & 7 & 33 & & \\
\hline Missing $^{\mathrm{a}}$ & 0 & & & & \\
\hline Clinical stage & & & & 0.497 & 0.781 \\
\hline I & 16 & 3 & 13 & & \\
\hline II & 139 & 24 & 115 & & \\
\hline III-IV & 81 & 11 & 70 & & \\
\hline Missing $^{\mathrm{a}}$ & 6 & & & & \\
\hline Size $(\mathrm{cm})$ & & & & 1.158 & 0.560 \\
\hline$<2$ & 41 & 7 & 34 & & \\
\hline$\geq 2$ & 199 & 31 & 168 & & \\
\hline Missing $^{\mathrm{a}}$ & 2 & & & & \\
\hline Lymph node & & & & 0.057 & 0.811 \\
\hline Negative & 93 & 14 & 79 & & \\
\hline Positive & 141 & 22 & 117 & & \\
\hline Missing $^{\mathrm{a}}$ & 8 & & & & \\
\hline ER stage & & & & 1.518 & 0.218 \\
\hline ER (-) & 91 & 17 & 74 & & \\
\hline ER $(+)$ & 134 & 17 & 117 & & \\
\hline Missing $^{\mathrm{a}}$ & 17 & & & & \\
\hline PR stage & & & & 0.033 & 0.857 \\
\hline PR (-) & 122 & 19 & 103 & & \\
\hline PR (+) & 102 & 15 & 87 & & \\
\hline Missing $^{\mathrm{a}}$ & 18 & & & & \\
\hline Her-2 stage & & & & 2.741 & 0.098 \\
\hline Her-2 (-) & 165 & 21 & 144 & & \\
\hline Her-2 (+) & 60 & 13 & 47 & & \\
\hline Missing $^{\mathrm{a}}$ & 17 & & & & \\
\hline Ki-67 stage & & & & 6.790 & $0.009^{\mathrm{b}}$ \\
\hline$<14 \%$ & 114 & 20 & 94 & & \\
\hline$\geq 14 \%$ & 46 & 1 & 45 & & \\
\hline Missing $^{\mathrm{a}}$ & 82 & & & & \\
\hline
\end{tabular}

${ }^{\mathrm{a}}$ Missing, samples were missing some medical records. ${ }^{\mathrm{b}} \mathrm{P}<0.01$. Neg., negative; Pos., positive. Amot, Angiomotin.

the number of invading and migrating Amot knockdown cells were significantly reduced, when compared to those of NC cells (Fig. 4B and C), suggesting that Amot downregulation 

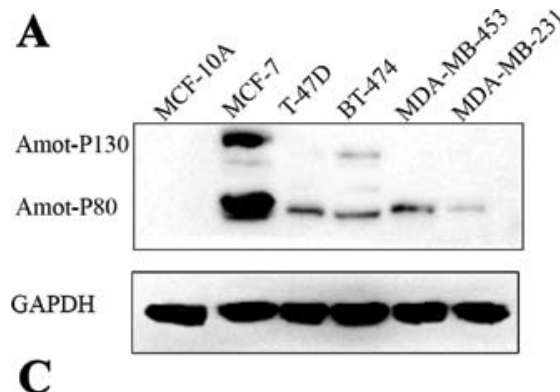

C

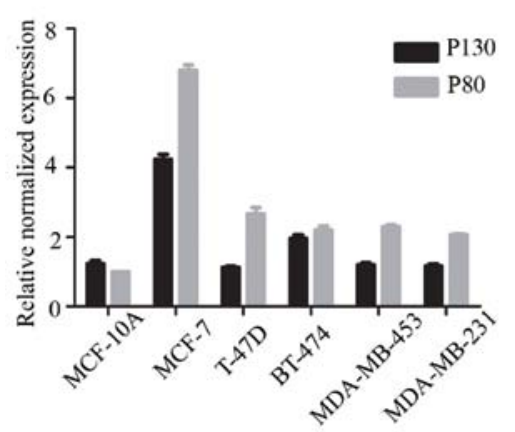

B

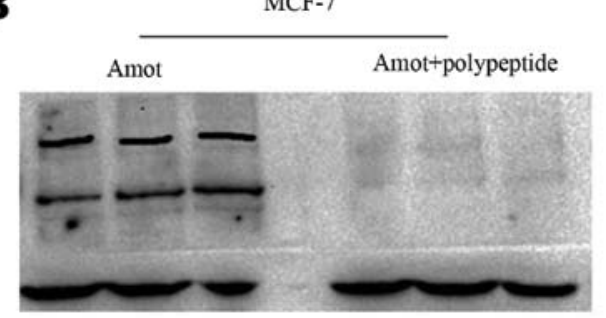

D
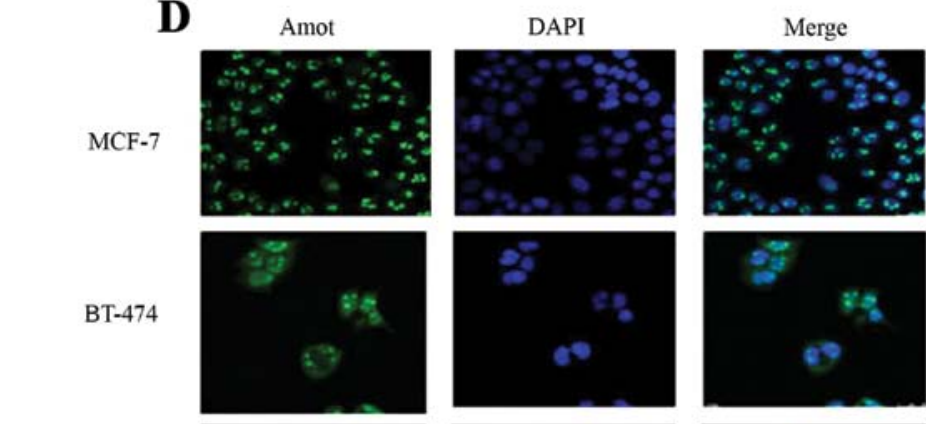

MDA-MB-231
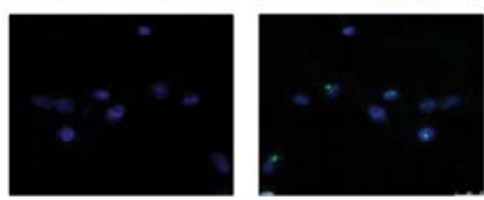

Figure 2. Expression and localization of Angiomotin (Amot) in breast cancer cells. (A) Western blot analysis showed Amot expression in breast cancer cells and mammary epithelial cells. GAPDH was used as an internal control. (B) Western blotting detected the antibody specificity in MCF-7 cells. The expression of Amot and Amot with polypeptide in MCF-7 cells. GAPDH was used as an internal control. (C) RT-PCR analyses showed Amot expression in breast cancer cells and mammary epithelial cell. The comparative CT method was used to quantify the relative levels of Amot mRNA. (D) Immunofluorescence assay shows Amot positively located in the nucleus in breast cancer cells.

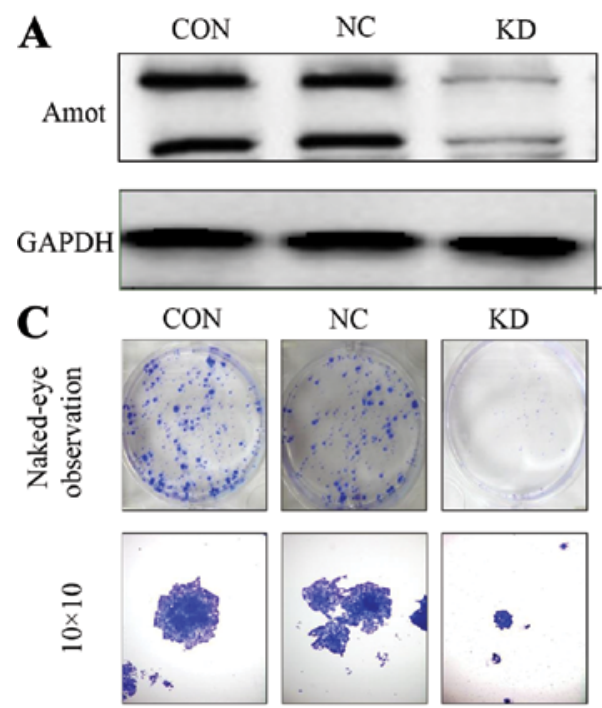

$\mathbf{E}$
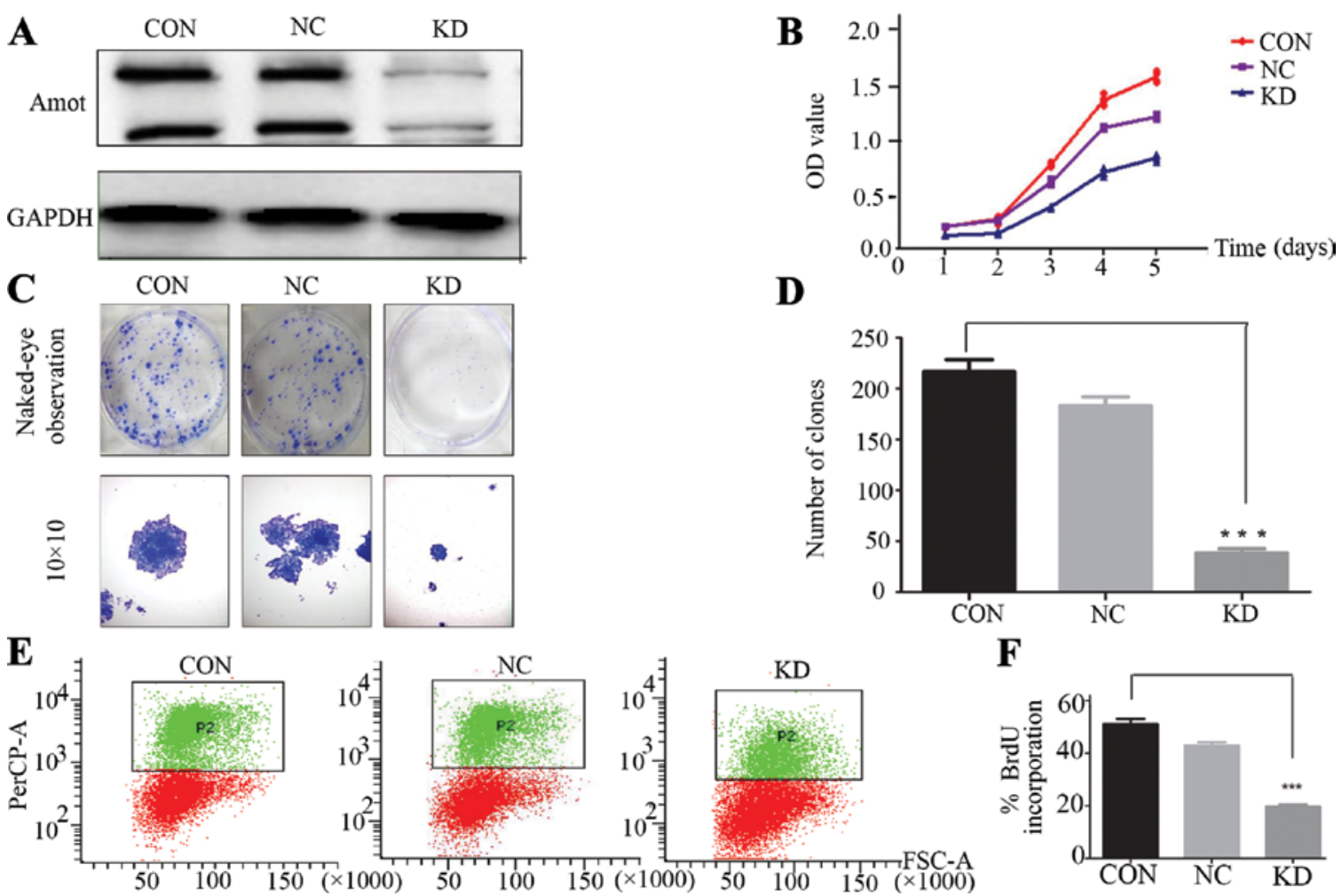

D
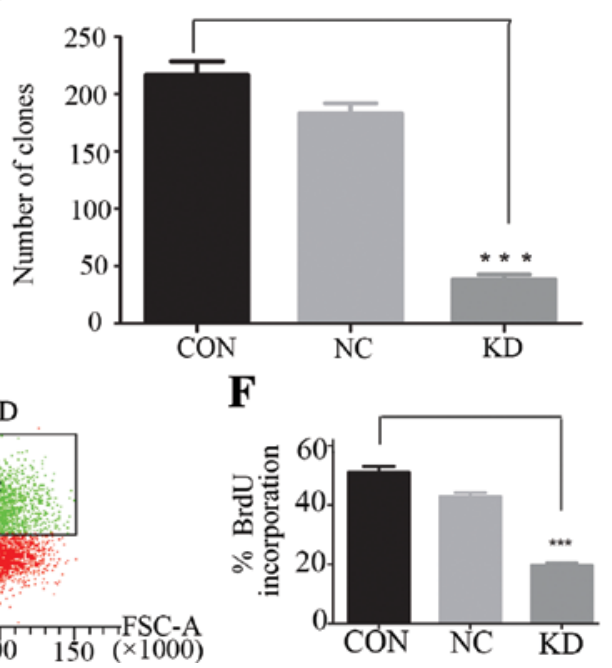

Figure 3. Downregulation of Angiomotin (Amot) decelerated the proliferation of MCF-7 breast cancer cell. CON (control), the uninfected breast cancer cells; $\mathrm{KD}$ (knockdown), the cells infected with the Amot shRNA lentivirus; NC (negative control), the mock control lentivirus. (A) Western blotting verificate knockdown efficiency of Amot in MCF-7 cells. GAPDH was used as an internal control. (B) MTT assays were performed to quantify relative numbers of MCF-7 cells at the indicated time points (1-5 days). (C) Plate clone formation assay detected the ability of MCF-7 cells forming clone in vitro by naked-eye observation or at 10x10 magnification. (D) The number of clones was quantified by counting cells in 10 random fields. (E) Brdu (5-bromodeoxyuridine) incorporation assay. Flow cytometry tested the number of Brdu incorporation in MCF-7 cells. (F) The percentage of Brdu incorporation in MCF-7 cells was quantitated to value the influence of Amot silence on the proliferation of breast cancer cells. The data represent the means $\pm \mathrm{SD} .{ }^{* * * *} \mathrm{P}<0.001$, compared to KD groups. 
$\mathbf{A}$
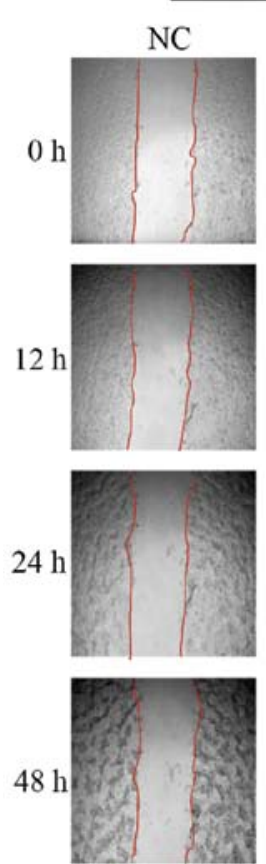
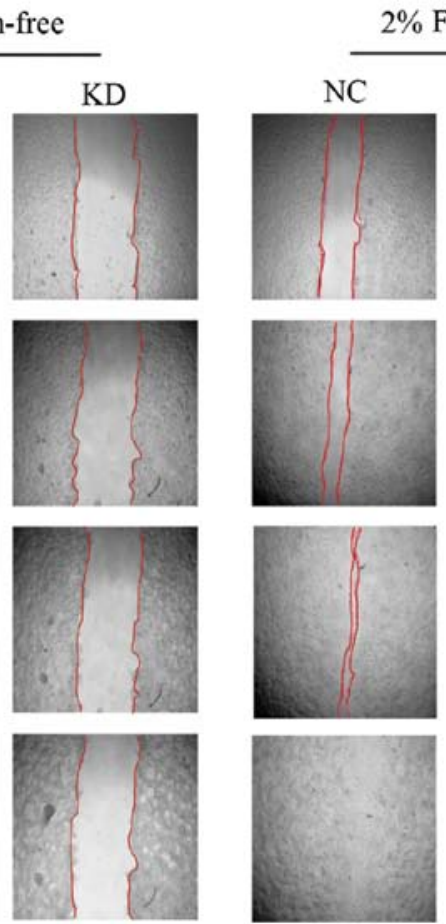

KD
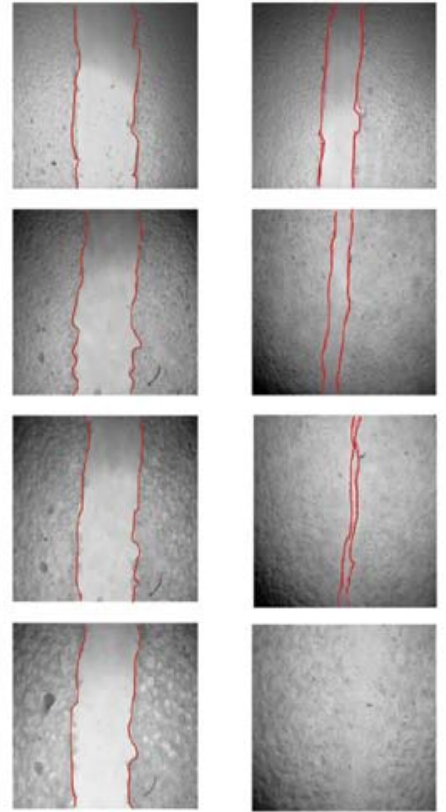

B

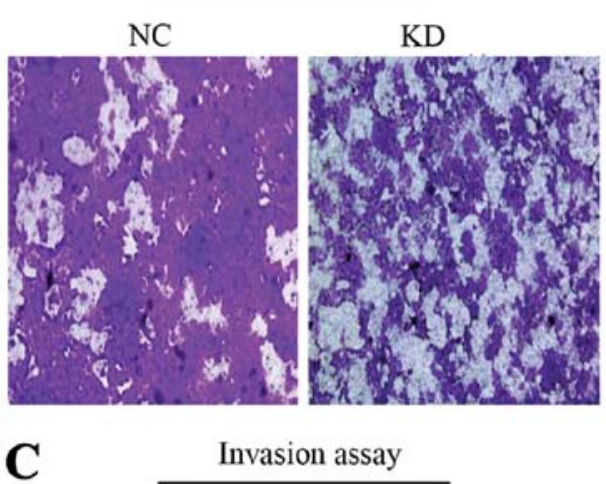

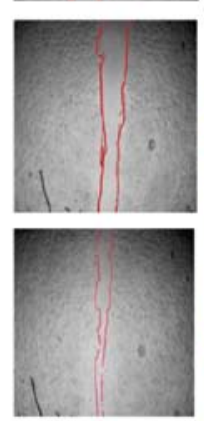

$\mathrm{KD}$
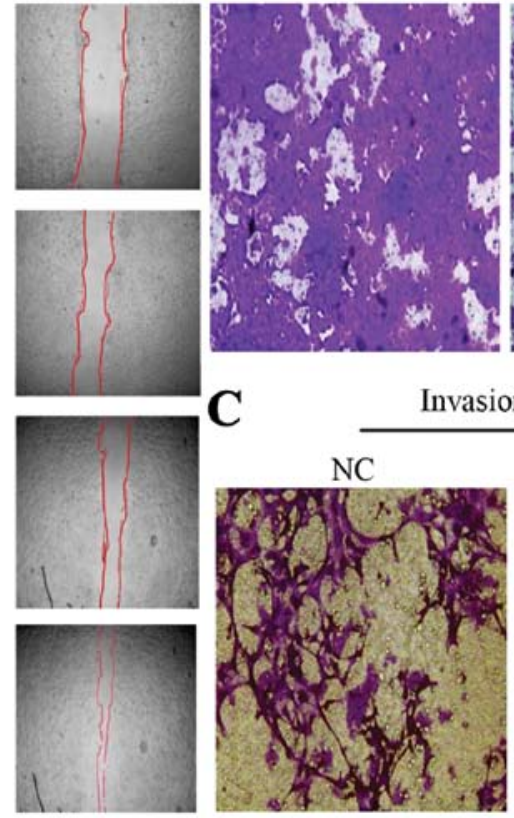

Migration assay

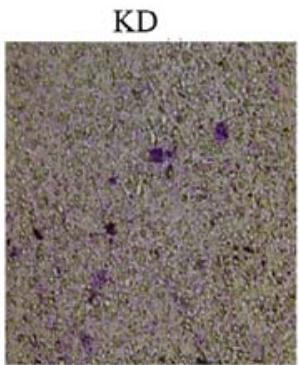

Figure 4. Downregulation of Angiomotin (Amot) decreased the capacity of invasion and metastasis of MCF-7 cells in vitro. CON (control), the uninfected breast cancer cells; KD (knockdown), the cells infected with the Amot shRNA lentivirus and; NC (negative control), the mock control lentivirus. (A) Wound-healing assay measured the effect of Amot downregulation on migration in MCF-7 cells. Cell migration into the wound area was photographed at indicated time points $(0-48 \mathrm{~h})$. (B) The migration assay measured the effect of Amot downregulation on migration in MCF-7 cells. Migration cells on the bottom surface of the filter were stained and photographed. (C) Invasion assay measured the effect of Amot downregulation on invasion in MCF-7 cells. Invading cells on the bottom surface of the filter were stained and photographed.

significantly decelerated the invasion and migration of MCF-7 cells in vitro.

Our results revealed that Amot knockdown significantly decreased the proliferative, invasive and metastatic capacity of MCF-7 cells in vitro.

Association of Amot and Hippo-YAP pathway. Amot has been shown to participate in the activities of the Hippo-YAP pathway (15). To elucidate the relationship of Amot and Hippo-YAP pathway, we examined the expression of YAP, YAP/TAZ, LATS1, MOB, MST1 and SAV1 in MCF-7 cells following the downregulation of Amot using western blot analysis. The results showed that the expression of YAP, YAP/TAZ and LATS1 was significantly decreased in MCF-7 cells following the knockdown of Amot (Fig. 5A). However, the expression of MOB, MST1 and SAV1 did not exhibit any notable change. Additionally, the expression of YAP was obviously decreased in the nucleoprotein (Fig. 5B and C).

\section{Discussion}

Amot is an angiostatin-binding protein that promotes endothelial cell migration and angiogenesis (5) and is expressed as the protein isoforms, p80-Amot and p130-Amot. The angiostatin-responsive migration-promoting function has been observed in the Amot p80 splicing variant, but not in the YAP-binding p130 variant (11). YAP-binding p130 Amot has been found to be involved in tumorigenesis. It was observed for the first time in 2011 that AmotL2 knockdown can activate YAP and induce cell transformation of MDCK epithelial cells, suggesting that Amot family proteins may play tumor suppressive roles (15). However, using RT-PCR Jiang et al (14) found that breast cancer tissues expressed significantly higher levels of Amot transcript, compared with normal mammary tissues. The Amot expression was significantly increased with the increasing degree of invasion and metastasis. Amot expression showed close relationships with VE-cadherin and PECAM-1. Findings of Jiang et al suggested that Amot was closely related to angiogenesis, invasiveness and poor survival of breast cancer (14). In 2008, Levchenko found that a DNA vaccine targeting Amot induced an antibody response and significantly inhibited angiogenesis and tumor growth (16). In addition, it has been reported that Amot expression likely promotes cell growth by prolonging the activation of MAPK signaling. Amot expression enhanced the proliferation rate of MCF-7 cells and induced MCF-10A cells to form large, disorganized spheroids in Matrigel. On the other hand, a reduced expression of Amot resulted in decreased ERK1/2-associated growth of MDA-MB-468 and SKBR3 cells (17). The above studies have indicated that Amot acts as a potential tumor promoter, which was well supported by our results.

In the present study, Amot was highly expressed in breast cancer tissues and cells, but weakly expressed in normal controls, while the expression level of Amot was increased in specimens from patients with a high level of Ki-67. The results indicated that Amot may be involved in breast cancer proliferation and invasion. Amot knockdown, not only retarded growth and viability of MCF-7 cells, but also significantly decreased the percentage of S-phase cells. In addition, lentiviral-mediated Amot silencing significantly reduced the 
A

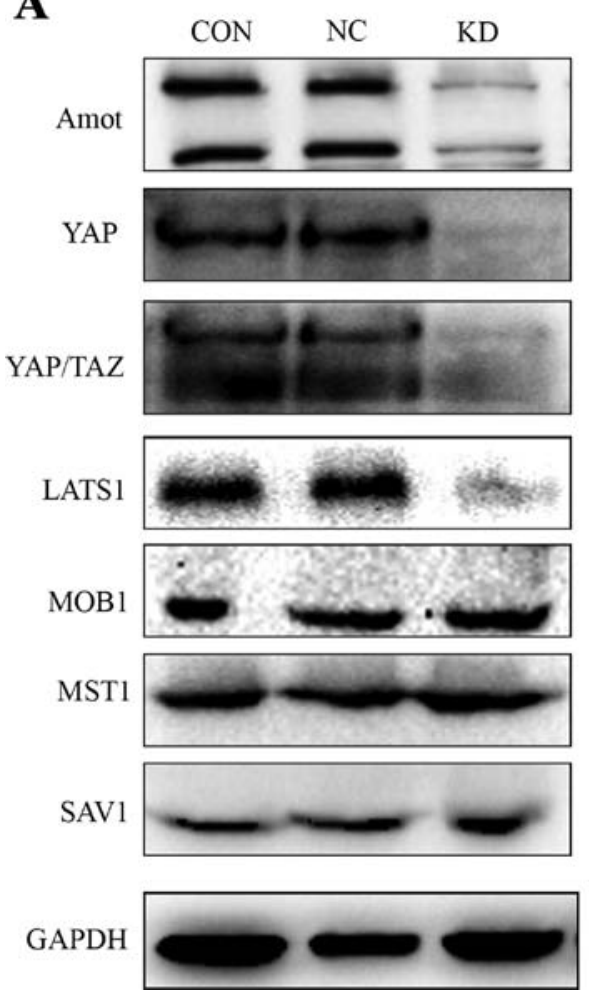

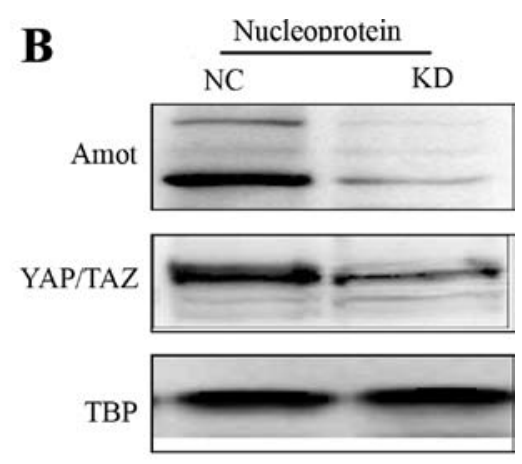

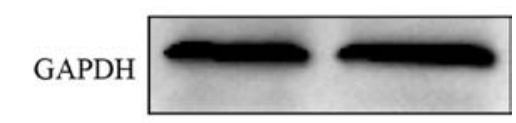

C Cytoplasmin protein
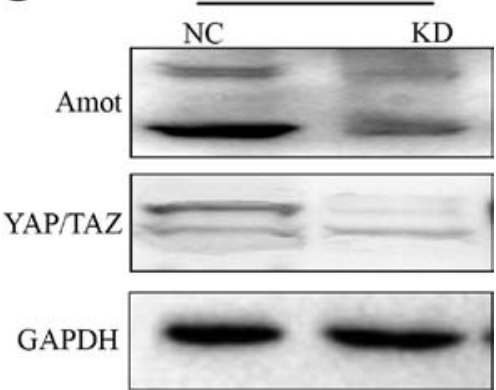

Figure 5. Preliminary study on Angiomotin (Amot) gene expression with Hippo-YAP signaling pathway. CON (control), the uninfected breast cancer cells; KD (knockdown), the cells infected with the Amot shRNA lentivirus; NC (negative control), the mock control lentivirus. (A) Western blotting detected the expression of Hippo-YAP signaling pathway protein (YAP, YAP/TAZ, LATS1, MOB, MST1 and SAV1) following the downregulation of Amot in MCF-7 cells. GAPDH was used as an internal control. (B) Western blotting was applied to detect the expression of Amot and YAP/TAZ in the nucleus after the downregulation of Amot in MCF-7 cells. GAPDH was used as an internal control and TBP was used as a nuclear protein marker. (C) Western blotting was applied to detect the expression of Amot and YAP/TAZ in the cytoplasm after the downregulation of Amot in MCF-7 cells. GAPDH was used as an internal control.

number of colonies formed by MCF-7 cells. Our results show that Amot downregulation inhibited MCF-7 cell proliferation. We also found that Amot knockdown in cells retarded the migration and invision of MCF-7 cells in vitro. Taken together, Amot downregulation decreased the proliferative, invasive and metastatic capacity of MCF-7 cells, indicating that Amot is a potential tumor promoter in breast cancer.

The Hippo-YAP signaling pathway regulates cell proliferation during development, tissue regeneration and carcinogenesis. Amot family proteins have been recently identified as negative regulators of YAP by promoting YAP phosphorylation to preventing their nuclear translocations (16-21). However, it has been shown that Amot-p130 may promote nuclear translocation of YAP and act as a transcriptional cofactor of the YAP-TEAD complex to facilitate biliary epithelial cell proliferation and liver cancer development either in response to tissue injury or in the absence of the tumor suppressor Merlin $(22,23)$. The different results were obtained probably since different organs and cells were used in those studies. In the present study, Amot knockdown significantly decreased the YAP and LATS1 expression in MCF-7 cells. Notably, the expression of YAP was obviously decreased in the nucleoprotein. The results suggest that Amot is involved in regulation of the proliferation of MCF-7 cells by modulating the nucleoprotein expression of YAP in the Hippo-YAP pathway. Future studies are needed to reveal the mechanism for a clear role of Amot in the Hippo-YAP pathway.

The respective roles of p80-Amot and p130-Amot were not studied in the present study since we did not make a clear distinction between p80 and p130 splicing variants of the Amot antibody and shRNA. Future studies are to focus mainly on the specific mechanisms of Amot and Hippo-YAP pathway, including the expression changes of total YAP, phosphorylated YAP, p80-Amot, and p130-Amot in the nucleus and cytoplasm, the activity of the downstream transcription factor TEAD, as well as the relationship between Amot and other signaling pathways associated with cell proliferation, invasion and metastasis.

In conclusion, our results have shown that Amot was highly expressed in breast cancer tissues and played an important role in promoting breast cancer cell proliferation and invasion. In addition, there was a more intimate connection between Amot and the Hippo-YAP pathway. Further studies are needed to reveal the mechanisms underlying the effect of Amot-induced Hippo-YAP pathway on breast cancer growth and invasion.

\section{Acknowledgements}

This study was supported by the National Natural Science Fund of China (no. 81172171). 


\section{References}

1. Siegel R, Ma J, Zou Z and Jemal A: Cancer statistics, 2014. CA Cancer J Clin 64: 9-29, 2014.

2. Jemal A, Bray F, Center MM, Ferlay J, Ward E and Forman D: Global cancer statistics. CA Cancer J Clin 61: 69-90, 2011.

3. Berry DA, Cronin KA, Plevritis SK, et al: Effect of screening and adjuvant therapy on mortality from breast cancer. $\mathrm{N}$ Engl J Med 353: 1784-1792, 2005.

4. Fong PC, Boss DS, Yap TA, et al: Inhibition of poly(ADP-ribose) polymerase in tumors from BRCA mutation carriers. N Engl J Med 361: 123-134, 2009.

5. Troyanovsky B, Levchenko T, Månsson G, Matvijenko O and Holmgren L: Angiomotin: an angiostatin binding protein that regulates endothelial cell migration and tube formation. J Cell Biol 152: 1247-1254, 2001.

6. Patrie KM: Identification and characterization of a novel tight junction-associated family of proteins that interacts with a WW domain of MAGI-1. Biochim Biophys Acta 1745: 131-144, 2005.

7. Levchenko T, Aase K, Troyanovsky B, Bratt A and Holmgren L: Loss of responsiveness to chemotactic factors by deletion of the C-terminal protein interaction site of angiomotin. J Cell Sci 116 : 3803-3810, 2003.

8. Ernkvist M, Aase K, Ukomadu C, et al: p130-Angiomotin associates to actin and controls endothelial cell shape. FEBS J 273 2000-2011, 2006.

9. Bratt A, Birot O, Sinha I, et al: Angiomotin regulates endothelial cell-cell junctions and cell motility. J Biol Chem 280: 34859-34869, 2005

10. Wells CD, Fawcett JP, Traweger A, et al: A Rich1/Amot complex regulates the $\mathrm{Cdc} 42$ GTPase and apical-polarity proteins in epithelial cells. Cell 125: 535-548, 2006.

11. Ernkvist M, Birot O, Sinha I, et al: Differential roles of p80- and p130-angiomotin in the switch between migration and stabilization of endothelial cells. Biochim Biophys Acta 1783: 429-437, 2008.
12. Ernkvist M, Luna Persson N, Audebert S, et al: The Amot/Patj/ Syx signaling complex spatially controls RhoA GTPase activity in migrating endothelial cells. Blood 113: 244-253, 2009.

13. Zheng Y, Vertuani S, Nyström S, et al: Angiomotin-like protein 1 controls endothelial polarity and junction stability during sprouting angiogenesis. Circ Res 105: 260-270, 2009.

14. Jiang WG, Watkins G, Douglas-Jones A, Holmgren L and Mansel RE: Angiomotin and angiomotin like proteins, their expression and correlation with angiogenesis and clinical outcome in human breast cancer. BMC Cancer 6: 16, 2006.

15. Zhao B, Li L, Lu Q, et al: Angiomotin is a novel Hippo pathway component that inhibits YAP oncoprotein. Genes Dev 25: 51-63, 2011.

16. Levchenko T, Veitonmäki N, Lundkvist A, et al: Therapeutic antibodies targeting angiomotin inhibit angiogenesis in vivo. FASEB J 22: 880-889, 2008.

17. Ranahan WP, Han Z, Smith-Kinnaman W, et al: The adaptor protein AMOT promotes the proliferation of mammary epithelial cells via the prolonged activation of the extracellular signal-regulated kinases. Cancer Res 71: 2203-2211, 2011.

18. Chan SW, Lim CJ, Chong YF, Pobbati AV, Huang C and Hong W: Hippo pathway-independent restriction of TAZ and YAP by angiomotin. J Biol Chem 286: 7018-7026, 2011.

19. Wang W,Huang J and Chen J: Angiomotin-like proteins associate with and negatively regulate YAP1. J Biol Chem 286: 4364-4370, 2011.

20. Dai X, She P, Chi F, et al: Phosphorylation of angiomotin by Lats1/2 kinases inhibits $\mathrm{F}$-actin binding, cell migration, and angiogenesis. J Biol Chem 288: 34041-34051, 2013.

21. Chan SW, Lim CJ, Guo F, Tan I, Leung T and Hong W: Actinbinding and cell proliferation activities of angiomotin family members are regulated by Hippo pathway-mediated phosphorylation. J Biol Chem 288: 37296-37307, 2013.

22. Hong W: Angiomotin'g YAP into the nucleus for cell proliferation and cancer development. Sci Signal 6: pe27, 2013

23. Yi C, Shen Z, Stemmer-Rachamimov A, et al: The p130 isoform of angiomotin is required for Yap-mediated hepatic epithelial cell proliferation and tumorigenesis. Sci Signal 6: ra77, 2013. 